\title{
Kidney macrophages: surveyors and scavengers of immune complexes
}

Small immune complexes (SICs) are thought to cause kidney damage by inducing type III hypersensitivity reactions, but the mechanisms involved are unclear. New findings show that macrophages residing in the kidney interact with peritubular endothelial cells to survey the transendothelial transport of proteins. Detection of SICs that trancytose into the interstitium triggers their uptake by kidney macrophages and induces an inflammatory response, indicating a direct role for these cells in initiating inflammation in response to SICs. "This observation provides a simple and novel model for the initiation of type III hypersensitivity in the kidney and identifies the kidney resident macrophage as a cell of interest in this type of disease," explains researcher Frederic Geissmann.

Type III hypersensitivity is a feature of several diseases that affect the kidney, including systemic lupus erythematosus and post- streptococcal glomerulonephritis. Previous work, demonstrating an involvement of macrophages in the scavenging of immune complexes, led Geissmann and colleagues to characterize resident macrophages in the kidney and their responses to immune complexes.

To determine the role of resident versus bone marrow-derived macrophages in regulating type III hypersensitivity, Geissmann and colleagues first assessed the ability of macrophages to scavenge fluorescently labelled albumin and SICs administered intravenously to mice. The researchers observed an accumulation of fluorescence within macrophages in the spleen, liver and kidney in a time and dosedependent manner, but no uptake by other macrophages, such as those of the dermis. Flow cytometry analysis showed that kidney macrophages take up SICs preferentially to albumin alone.

The findings of albumin and SIC uptake by spleen and liver macrophages is unsurprising, given that that the sinusoidal endothelium of the liver and red pulp of the spleen enables direct contact of macrophages with the bloodstream; however, the uptake of these particles by kidney macrophages was somewhat unexpected. Using intravital microscopy and transmission electron microscopy, Geissmann and colleagues found that kidney macrophages form a dense network in close association with the abluminal side of the endothelium of peritubular capillaries. Moreover, parabiosis experiments revealed that $<1 \%$ of kidney macrophages transfer between pairs, suggesting that the kidney macrophages are tissueresident, and not infiltrating or derived from the bone marrow. Further characterization demonstrated that unlike skin macrophages, kidney macrophages express the $\mathrm{F} c \gamma$ receptor, Fc $\gamma$ RIV.

Using ultrastructural analyses and intravital imaging, the researchers showed that intravenously administered antibodies and SICs enter the kidney interstitium through transendothelial transport in a process mediated by caveolae and clathrin-coated vesicles. Unlike liver and spleen macrophages, which phagocytose large particles and immune complexes, kidney resident macrophages were found to phagocytose only small particles of 20-200 nm. Geissmann and colleagues found that the uptake of SICs by kidney macrophages occurred in an Fc $\gamma$ RIV-dependent manner, leading to initiation of an inflammatory response with production of TNF, activation molecules, and the recruitment of monocytes and neutrophils. Intravenous administration of autoantibody-containing serum from lupus-prone mice also led to Fc $\gamma$ RIV-dependent activation of kidney macrophages, whereas administration of SICs containing GpC oligonucleotides indicated that nucleic acids activate a Tolllike receptor signalling pathway, which synergises with the Fc $\gamma$ RIVdependent pathway to stimulate resident macrophages.

The researchers are now planning further experiments to assess the importance of these processes in immunologic diseases of the kidney. "Immune complex-mediated diseases — such as lupus nephritis - are complex and chronic processes," says Geissmann. "We plan to investigate the role of resident macrophages in their pathogenesis, to test whether manipulation of resident macrophages can improve the outcome and to characterize molecular therapeutic targets."

\section{Susan J. Allison}

ORIGINAL ARTICLE Stamatiades, E. G. et al. Immune monitoring of trans-endothelial transport by kidney-resident macrophages. Cell http:// dx.doi.org/10.1016/j.cell.2016.06.058 (2016) 